\title{
Effect of creep on the Bauschinger effect in a polycrystalline austenitic stainless steel
}

\author{
J.N. Hu ${ }^{\mathrm{a}}$, A.C.F. Cocks ${ }^{\mathrm{a}}$ \\ ${ }^{a}$ Department of Engineering Science, University of Oxford, OX1 3PJ, UK
}

\begin{abstract}
A combined thermodynamic/kinetic approach is adopted to derive the climbcontrolled recovery of dislocation junctions on individual slip planes in a material during creep when dislocation core diffusion is the rate controlling kinetic process. The model is used in an integrated multi-scale crystal plasticity/creep self-consistent framework to evaluate the effect of creep pre-strain on the Bauschinger effect in a polycrystalline austenitic stainless steel. The model predictions are in good agreement with experimental findings of the effect of tensile creep on the subsequent low temperature response in tension and compression and on the development of residual stresses determined from neutron diffraction studies.
\end{abstract}

Keywords: Bauschinger effect; Creep recovery; Self-consistent model; Thermodynamics

A complete physical understanding of creep deformation mechanisms across different length-scales is critical for the development of mechanistic models for the prediction of component performance in the power generating industry, which may be subject to complex thermo-mechanical loading conditions $[1,2]$. This requires knowledge of how the material internal state and/or microstructure evolve with deformation [3].

Pre-strained materials exhibit Bauschinger effect, an important manifestation of the material kinematic feature [3], which has been proposed to be related to the internal stress developed during forward loading and how this influences dislocation movement when the load is reversed [4]. However, a recent review pointed out the ambiguous definition of "internal stress" in literature and classified the internal state 
into internal resistance and internal stress to provide a more meaningful mechanistic description [3]. Internal resistance is essentially a property, referring to the intrinsic resistance to dislocation motion and thereby the inelastic deformation, thus is not "stress" but "strength". In contrast, internal stress is the residual stress which maintains the material internal self-equilibrium $[3,5]$.

Multi-scale crystal plasticity self-consistent models [5, 6], developed from the length-scale of elementary slip systems to predict the bulk response, characterise the internal resistance in terms of the critical resolved shear strength (CRSS) on the individual slip systems, and the residual stress as a consequence of plastic mismatch (anisotropy) between grains at the meso-scale, as well as interactions between the matrix, dislocations and precipitates at the micro-scale [5]. The model takes into account forest dislocation hardening described by the evolution of the number density of forest dislocation junctions (or dislocation segments between junctions), as well as additional strengthening effects arising from solid solution elements and precipitates. The key state variable is the mean spacing between different types of obstacles, which is inversely proportional to the respective contributions to the CRSS. Dislocation multiplication during deformation introduces more segments and junctions, leading to a decrease of mean spacing and an increase of CRSS on a slip plane. Details of the model can be found in [5-8].

The model has provided a physical interpretation of room temperature experiments of lattice deformation measured by in-situ neutron diffraction [6,9] and the macroscopic Bauschinger effect of plastically pre-strained Type 316H F.C.C. polycrystalline stainless steel $[5,10]$. As the temperature increases, the kinematics of inelastic deformation may change, where dislocations can recover by climb-controlled network coarsening, a process whereby long segments grow at the expense of shorter ones, leading to a reduction of segments/junctions and an increase in mean spacing, opposite to the multiplication process. Such a process has been modeled by Lagneborg [11] and many other researchers [12-14] as analogous to grain growth [15]. Such an analogy might be appropriate for situations where lattice diffusion controls recovery, but is not appropriate for situations where dislocation core diffusion or pipe diffusion is the dominant transport mechanism, which is of practical interest in austenitic stainless steels [16-19]. In this paper we employ the thermodynamic variational principle described by Cocks et al [20] to derive a simple climb-controlled recovery law for the distribution of dislocation segments/junctions on individual slip 
planes, which captures the major physical processes. This is combined with our previous model described above to provide a fully coupled creep/plasticity model. We employ the model to evaluate the effect of creep pre-strain history on the Bauschinger effect of Type $316 \mathrm{H}$ stainless steel.

Consider the variational functional

$$
\Pi=\Psi+\dot{\Phi}
$$

where $\Psi$ is a rate potential related to the dominant kinetic processes (dislocation core diffusion) and $\dot{\Phi}$ is the rate of change of Gibbs free energy (total dislocation line energy). Cocks et al [20] demonstrate that the best approximation for the rate of evolution of the microstructure (mean spacing of dislocation junctions in the current context) within the range of idealized geometries considered is that which minimizes the functional $\Pi$ (i.e. $\delta \Pi=0$ ).

First, we identify a thin slab of material with thickness $d z$ (Fig. 1a). Consider $N_{d}$ as the number of junctions per unit area on a slip plane ( $x-y$ plane in Fig. 1a). Assume that these junctions are associated with dislocation segments of uniform length $d z$ within the slab. $N_{d} \times d z$ is the total length in the volume $d z \times 1$. In F.C.C materials, the total dislocation density $(\rho)$ can be defined as the arithmetic mean of the contributions from the four families of slip planes:

$$
\rho=\frac{1}{4}\left(N_{d 1}+N_{d 2}+N_{d 3}+N_{d 4}\right) \cdot \frac{d z}{d z \times 1}=\frac{1}{4}\left(\frac{1}{{L_{d 1}}^{2}}+\frac{1}{{L_{d 2}}^{2}}+\frac{1}{L_{d 3}{ }^{2}}+\frac{1}{{L_{d 4}}^{2}}\right)
$$

where $L_{d i}=N_{d i}^{-1 / 2}$ is the mean spacing of junctions on $i$ th slip plane. Therefore, $\dot{\Phi}$ is calculated as

$$
\dot{\Phi}=\dot{\rho} \Gamma=-\frac{1}{2} \Gamma\left(\frac{1}{L_{d 1}^{3}} \dot{L}_{d 1}+\frac{1}{L_{d 2}^{3}} \dot{L}_{d 2}+\frac{1}{L_{d 3}^{3}} \dot{L}_{d 3}+\frac{1}{L_{d 4}^{3}} \dot{L}_{d 4}\right)
$$

where $\Gamma=1 / 2 G b^{2}$ is the dislocation line energy ( $G$ : shear modulus; $b$ : magnitude of Burgers vector). 


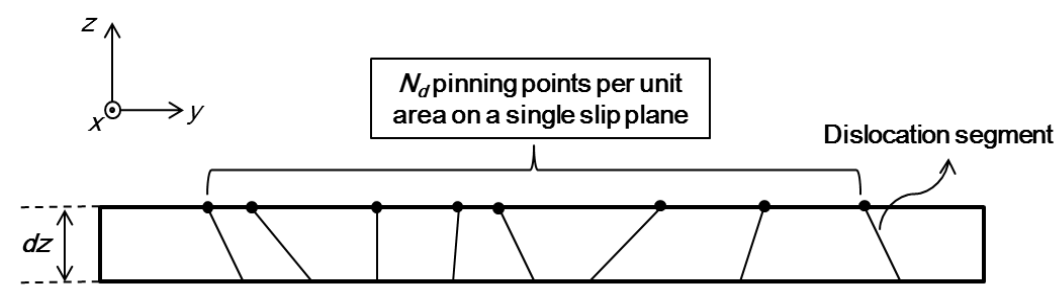

(a)

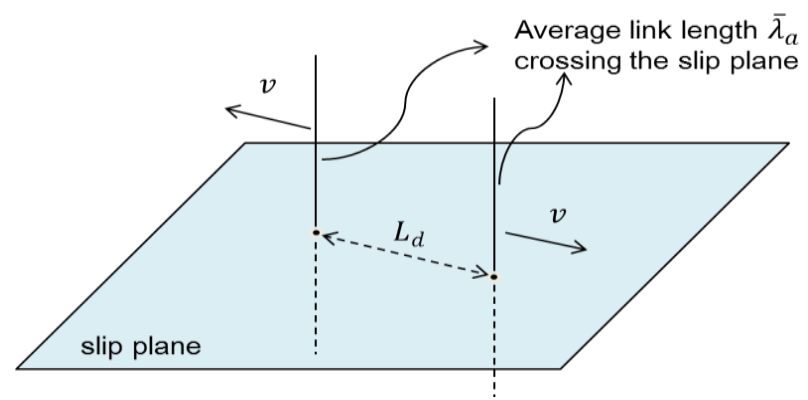

(b)

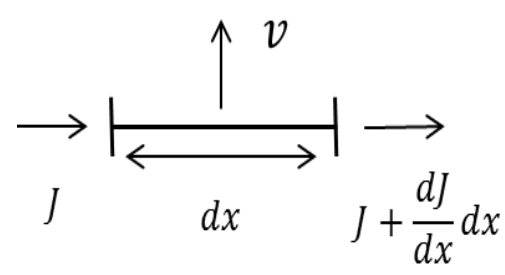

(c)

Fig 1. (a) A thin slab containing $N_{d} x d z$ length of dislocation segments; (b) Schematic of climbing (at a uniform rate $v$ ) of two segments crossing a slip plane; (c) Diffusional flux across a small length $d x$ on a segment by dislocation core diffusion

The kinetic process of network coarsening indicates that junctions can move as a result of glide, cross-slip or climb of dislocation segments. We make the simplified assumption that dislocations glide freely and energy is only dissipated as a result of climb, thus the creep rate is controlled by climb as discussed by Sherby and Weertman [21]. It is simply assumed that half the segments climb while the other half glide or cross-slip. Now consider two segments crossing a single slip plane (Fig. 1b) with an average length $\bar{\lambda}_{a}$ and separation $L_{d}$. Assume that atoms predominantly diffuse through the dislocation core and the segment climbs at a uniform rate $v$ along the length. Consider an elemental length $d x$ on the segment, as shown in Fig. 1(c). The magnitude of the rate of volume of atoms flowing into or out of $d x$ is

$$
\frac{d V}{d t}=\left|\frac{d J}{d x}\right| d x \cdot a_{c}=|v| \cdot d x \cdot b
$$

where $a_{c}$ is the core area and $J$ is the volumetric flux, defined as the volume of atoms flowing through unit area per unit time. Note that $v \approx \dot{L}_{d} / 2$ in terms of Fig. 1(b), Eq. (4) then determines $J$ 


$$
|J|=\frac{|v| b}{a_{c}} x+W=\frac{\left|\dot{L}_{d}\right| b}{2 a_{c}} x+W
$$

$W$ is an integration constant. Eq. (5) indicates that the flux varies along the segment. The average flux $\bar{J}$ can be simply calculated as (assume $J=0$ at the center of the segment, i.e. $W=0$ at $x=0)$.

$$
|\bar{J}|=\frac{2}{\bar{\lambda}_{a}} \int_{0}^{\bar{\lambda}_{a} / 2}|J| d x=\frac{\left|\dot{L}_{d}\right| b \bar{\lambda}_{a}}{8 a_{c}}
$$

According to Fick's law, $J=M f$, where $M=D_{c} \Omega / k T$ is the climb mobility $\left(D_{c}\right.$ : core diffusivity; $\Omega \approx b^{3}$ : atomic volume; $k$ : Boltzmann constant and $T$ : temperature) and $f$ is the thermodynamic driving force for diffusion - the negative of the chemical potential gradient. Note that the total length of the climbing segments per unit volume crossing the slip plane is of the order of $1 / \bar{\lambda}_{a}^{3} \times \bar{\lambda}_{a}=1 / \bar{\lambda}_{a}^{2}$ (it is not necessary to determine the detailed proportionality here; this will be addressed later). The total volume $V$ of the climbing segments per unit volume can then be written as $V=a_{c} / 2 \bar{\lambda}_{a}^{2}$ (only half segments climb). Therefore, the rate potential $\Psi_{a}$ for the climbing segments on this slip plane can be determined by

$$
\Psi_{a}=\int_{V} \frac{1}{2} f \cdot J d V=\int_{V} \frac{1}{2 M} J^{2} d V=\frac{D_{c} b^{3}}{2 k T} \bar{J}^{2} \cdot \frac{a_{c}}{2 \bar{\lambda}_{a}^{2}}
$$

Taking into account all the four slip planes, the overall rate potential $\Psi$ can be obtained, considering Eq. (6), as

$$
\Psi=\sum \Psi_{a}=\frac{D_{c} b^{3}}{512 k T}\left(\dot{L}_{d 1}^{2}+\dot{L}_{d 2}{ }^{2}+\dot{L}_{d 3}{ }^{2}+\dot{L}_{d 4}{ }^{2}\right)
$$

where $a_{c}$ is taken as $2 b^{2}$ [16]. Eq. (8) shows that $\Psi$ is independent of the precise definition of $\bar{\lambda}_{a}$. Eqs. (3) and (8) constitute the functional $\Pi$ in Eq. (1) and each $\dot{L}_{d i}$ (and $\dot{N}_{d i}$ accordingly) can be obtained by noting that the stationary value of $\Pi$ is when $\partial \Pi / \partial \dot{L}_{d 1}=\partial \Pi / \partial \dot{L}_{d 2}=\partial \Pi / \partial \dot{L}_{d 3}=\partial \Pi / \partial \dot{L}_{d 4}=0$, which is independent of $\dot{L}_{d i}$ on other slip planes:

$$
\dot{L}_{d i}=\frac{64 D_{c} G b^{5}}{k T} \cdot \frac{1}{L_{d i}{ }^{3}} \rightarrow \frac{W_{c} D_{c} G b^{5}}{k T} \cdot \frac{1}{L_{d i}{ }^{3}} \quad(i=1 \sim 4)
$$

Here we have replaced the constant 64 with a fitting parameter $W_{c}$ to compensate for 
some assumptions described above, such as the simplified calculation of the average flux $\bar{J}$ on each climbing segment (Eq. 6) as well as the number of climbing segments. Creep is understood to be a competition between hardening and recovery. Taking into account our previous forest dislocation hardening model $[6,7]$, an overall evolution law of $L_{d i}$ on $i$ th slip plane can be expressed as

$$
\dot{L}_{d i}=-\frac{1}{2} L_{d i}{ }^{3} \cdot\left(j_{s} \dot{\gamma}_{i}+j_{l} \sum_{k \neq i} \dot{\gamma}_{k}\right)+\frac{W_{c} D_{c} G b^{5}}{k T} \cdot \frac{1}{L_{d i}{ }^{3}}
$$

where $\dot{\gamma}_{i}$ refers to the plastic shear strain rates on $i$ th slip plane. The physics of the interaction between hardening on different slip planes is controlled by the use of two fitting parameters $j_{s}$ (self-hardening) and $j_{l}$ (latent hardening) [6, 7]. Note that as a direct consequence of using an arithmetic mean to determine the total dislocation density $\rho$ (Eq. 2), there is no interaction between recovery on different slip planes. Other choices, such as overlap of dislocation segments between different slip planes, may result in non-linear approximations of $\rho$ and lead to changes in the final relationship in Eq. (9). However, these are not explored here to avoid introducing more complexity into the model. Eq. (10) can be used to determine the evolution of the internal resistance, CRSS, on individual slip planes. During steady state, $\dot{L}_{d i} \approx 0$, i.e. a balance is reached between hardening and recovery.

Here the derived creep model is incorporated in our previous model $[5,6]$ and used to evaluate the Bauschinger effect of ex-service plus laboratory aged (EXLA, detailed description in [10]) Type $316 \mathrm{H}$ polycrystalline austenitic stainless steel after being subjected to a tensile short-term creep pre-strain at $250 \mathrm{MPa}$ at high temperature (HT, $550^{\circ} \mathrm{C}$ ). The tests have been interrupted at $\sim 180 \mathrm{~h}$ primary (designated EXLA3) or $\sim 1000$ h secondary stage (designated EXLA4) [22]. This extends our previous work [5] on the same samples which have only experienced time-independent plastic straining (or at the beginning of primary) at HT (designated EXLA2). After HT prestraining, each sample was air cooled by removing the insulation of the furnace while the load was still applied [22]. Additional samples crept at higher stresses as provided by EDF Energy are also evaluated here. All slip systems for all grains were assumed to have identical CRSS before commencement of any loading (no initial residual stress). The distribution and strengthening effect of precipitates and solid solution have been discussed in [5] and are assumed to be fixed, i.e. phase transformations 
(e.g. growth of precipitates) is ignored during the prescribed short-term creep. In each simulation the material starts with uniaxial $\mathrm{HT}$ pre-straining at $550^{\circ} \mathrm{C}$ up to the prescribed stress and is then allowed to creep for a prescribed period, followed by cooling/unloading (the developed CRSS and residual stress remain but scale with the temperature-dependent shear modulus $[16,23])$ and subsequent reloading at room temperature (RT) either in tension (designated EXLA2-1, EXLA3-1 and EXLA4-1) or compression (designated EXLA2-2, EXLA3-2 and EXLA4-2). The HT tensile prestrain history and creep curves are used to calibrate the model. All parameters used in our previous work remain the same [5]. Two new parameters as derived in this study (Eq. 10) are needed to simulate creep, i.e. the core diffusivity $D_{c}$ and $W_{c}$. There is limited data on dislocation core diffusion compared with the extensive literature on self-diffusion. The dislocation core diffusivity is typically several orders of magnitude large than that for self-diffusion with an activation energy about 0.6 to 0.7 times that for self-diffusion [16, 24]. Here we use the diffusivity given by Frost and Ashby [16] and Evans [18] in our calculations $\left(D_{c}=\mathbf{6 . 4 7 \times 1 0 ^ { - 1 5 }} \mathrm{m}^{2} / \mathrm{s}\right)[16,18] . W_{c}=\mathbf{4 0}$ is determined by fitting the experimental creep curve.

The model prediction matches very well with the RT reloading tensile/compressive data (with creep pre-strain history at 250MPa) as shown in Fig. 2(a), thus principally captures the different amount of hardening and residual stress fields. The measured and fitted creep curves at 250MPa are shown separately in Fig. 3. For a comparison, measured and predicted results for EXLA2 specimens [5] are also shown in Fig. 2(a). All these data are extracted from [10], and all the compression data are shown using absolute values. The differences in the creep curves at 250MPa has been attributed to variability in the service history of each specimen [10] and considered to be acceptable due to the similar steady state creep rates. In Fig. 2(a), different yield stresses observed between the HT pre-straining curve and RT reloading curves is due to the way in which the CRSS scales with temperature. The sharper yield exhibited in all tensile reloading curves is attributed to a suitable residual stress field established by HT pre-straining thus all grains deform compatibly plastically during tensile reloading [5]. While the gradual yield exhibited in all reverse compressive reloading curves is attributed to significant residual stress redistribution in all grains as the material deforms plastically. Hu and Cocks [5] have identified a number of physical processes not included in the model that could affect the quality of the fit to the data. 
A potential source of error is the relaxation of residual stress and change of microstructure during cooling down after creep. For the fast rate of cooling employed in these experiments, we would expect both these effects to be small. This is supported by the observation that the theory captures a number of interesting trends that are observed experimentally, such as, with increasing creep strain, (1) there is a smoother yielding process under tensile loading, and (2) the compressive curve asymptotes more rapidly towards the tensile curve. It is also interesting to note that as the creep strain increases towards the steady state the tensile yield strength gradually increases. This is due to the evolving microstructure and residual stress state as the material creeps. The effect on the mechanical response of both these processes appears to be well captured by the model.

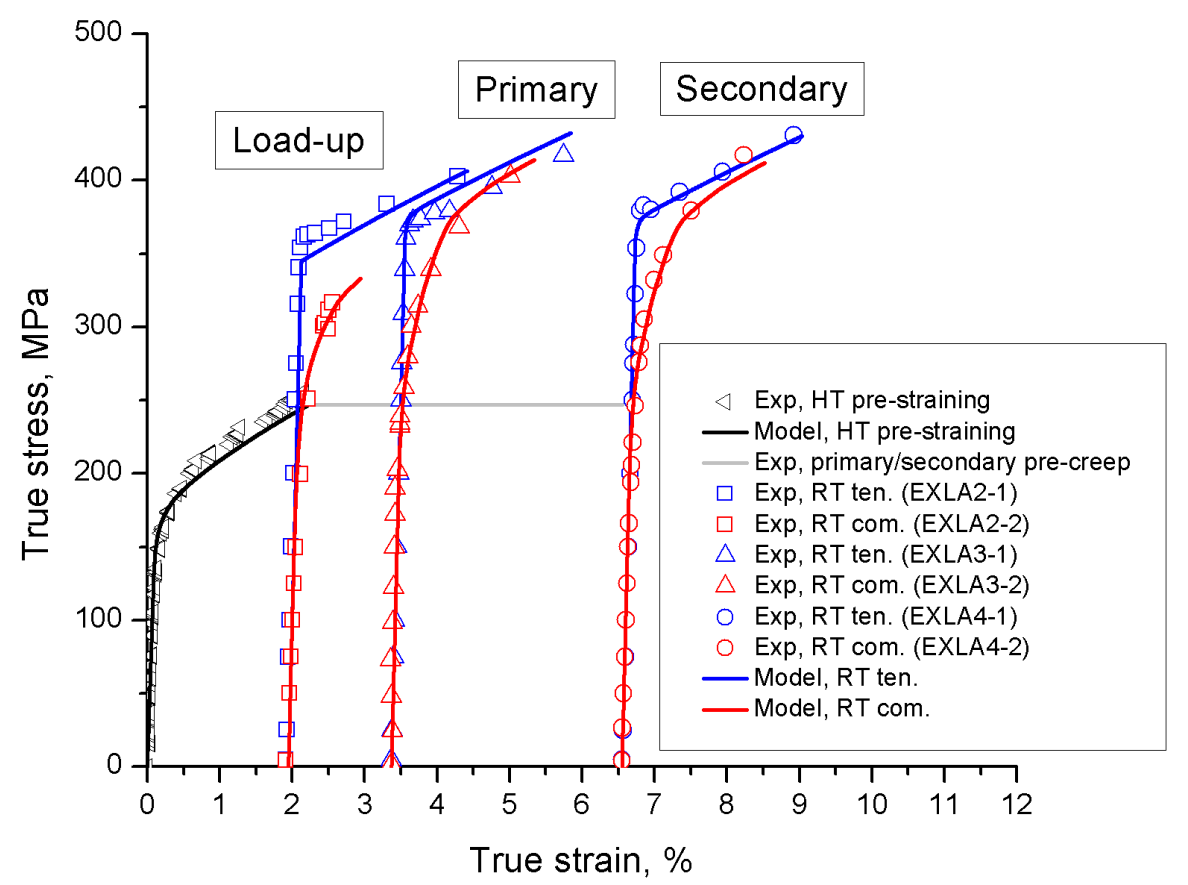

(a) 


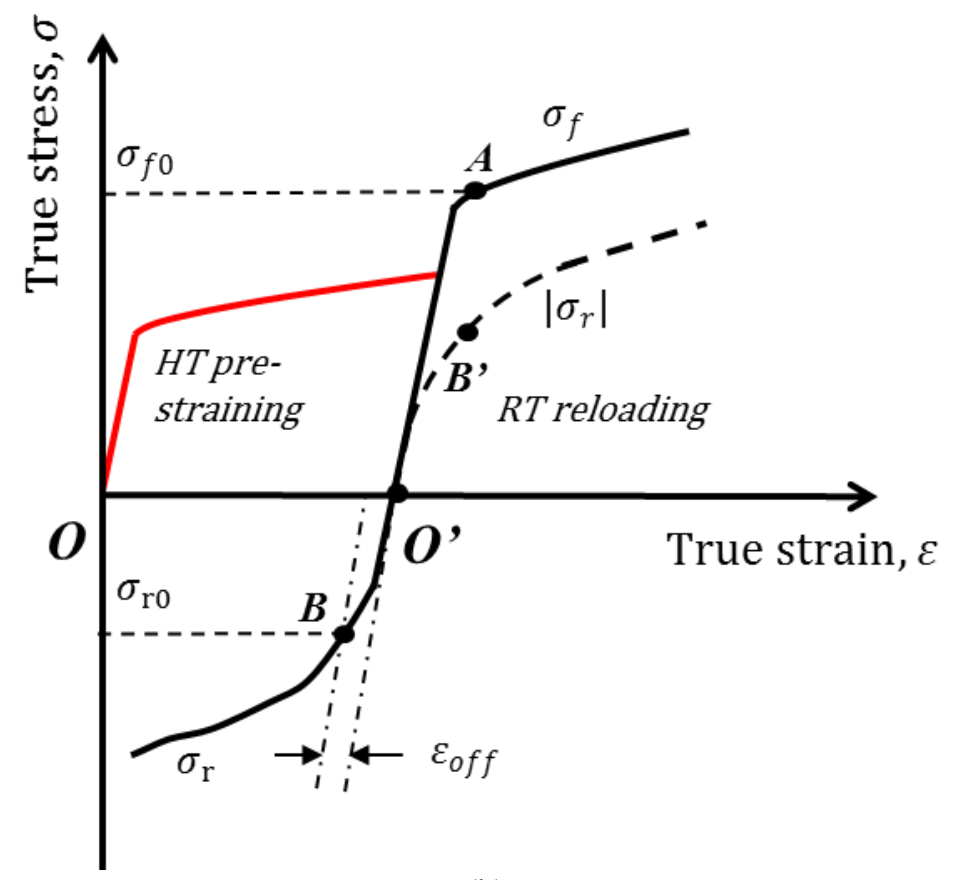

(b)

Fig 2. (a) Comparison between measurement and model prediction of high temperature (HT, $550^{\circ} \mathrm{C}$ ) tensile pre-straining and subsequent room temperature (RT) tensile/compressive reloading for pre-strained or pre-crept specimens. Data and prediction for compression is shown in absolute value. (b) Schematic of the Bauschinger test with stress variables and empirical strain offset

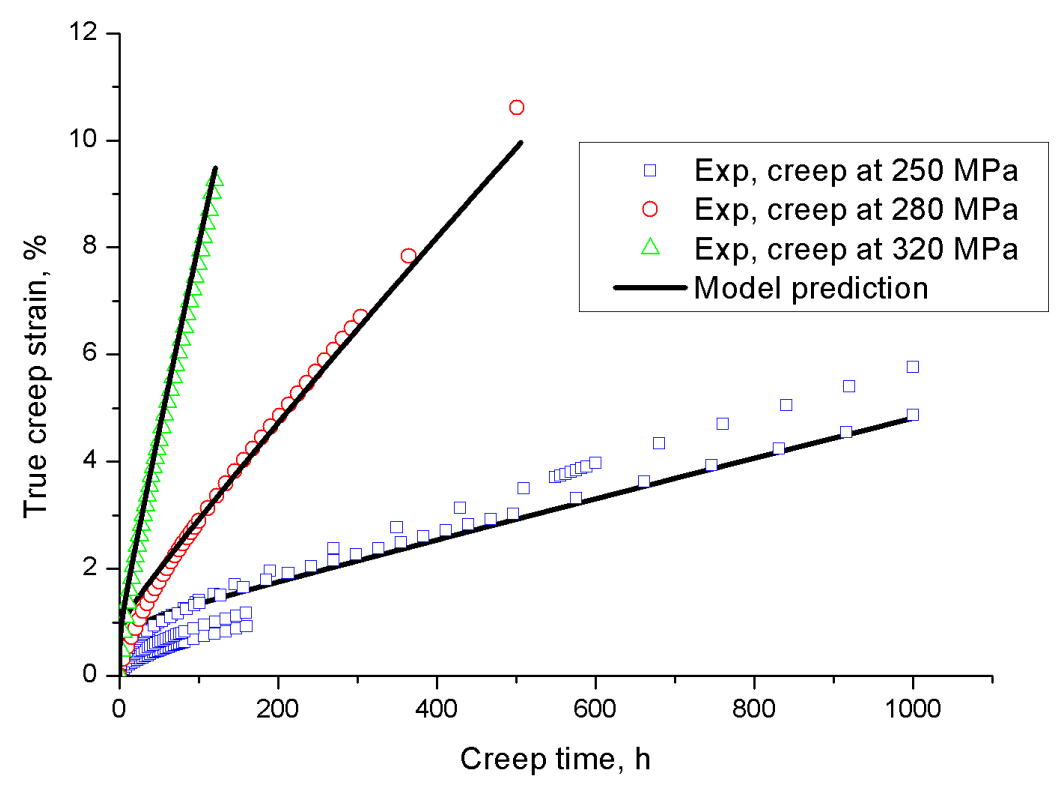

Fig 3. Measured and predicted creep curves at different stresses. Data at 250MPa consists of both primary and secondary creep curves.

Apart from the geometrical evaluation in Fig. 2(a), as an alternative, three empirical variables have been used in literature to mechanistically evaluate the Bauschinger effect [10]: (i) an initial yield stress, $\sigma_{0}$, describing an isotropic resistance to create inelastic flow; (ii) $\sigma_{\text {iso }}$ describing the contributions to isotropic 
hardening; and (iii) $\sigma_{k i n}$ describing the contributions to kinematic hardening. In the schematic and definition shown in Fig. 2(b), the flow stress at forward tensile loading $\sigma_{f}$ and reverse compressive loading $\sigma_{r}$ can be expressed as

$$
\left\{\begin{array} { l } 
{ \sigma _ { f } = \sigma _ { 0 } + \sigma _ { i s o } + \sigma _ { k i n } } \\
{ \sigma _ { r } = - \sigma _ { 0 } - \sigma _ { i s o } + \sigma _ { k i n } }
\end{array} \rightarrow \left\{\begin{array}{l}
\sigma_{i s o}=\frac{\sigma_{f}-\sigma_{r}}{2}-\sigma_{0} \\
\sigma_{k i n}=\frac{\sigma_{f}+\sigma_{r}}{2}
\end{array}\right.\right.
$$

For the material employed here, $\sigma_{0}$ has been determined from the reference specimens [10]. For pre-strained specimens, a small empirical strain offset, $\varepsilon_{\text {off }}=10^{-4}$, was used to determine the "initial" yield point $A(B)$ on each tensile (compressive) curve with the corresponding flow stress $\sigma_{f 0}\left(\sigma_{r 0}\right)$ as shown in Fig. 2(b). The predicted and experimentally calculated evolution of the two stress terms are shown in Fig. 4. Also shown in the figure are results for two additional specimens with different pre-crept histories subject to 280MPa and 320MPa. Predictions of the two additional creep curves are shown in Fig. 3 without any change in the parameters. Note that an extended period (still short-term) of creep for each specimen has been examined, as reflected from the enriched results in Fig. 4. As the stress increases, the model predicts an enhancement of both isotropic and kinematic stresses as well as the difference between the two terms $\left(\sigma_{i s o}-\sigma_{\text {kin }}\right)$. The latter trend demonstrates the enhancement of the compressive flow stress $\left|\sigma_{r 0}\right|$ (Eq. 12 with given $\sigma_{0}$ ), which in reality is reflected in Fig. 2(a). Further, both terms remain almost constant regardless of the extended period of secondary creep, which indicates the achievement of a steady state. This trend is also understood to be consistent, in an empirical way, with that of the internal resistance and residual stress. 


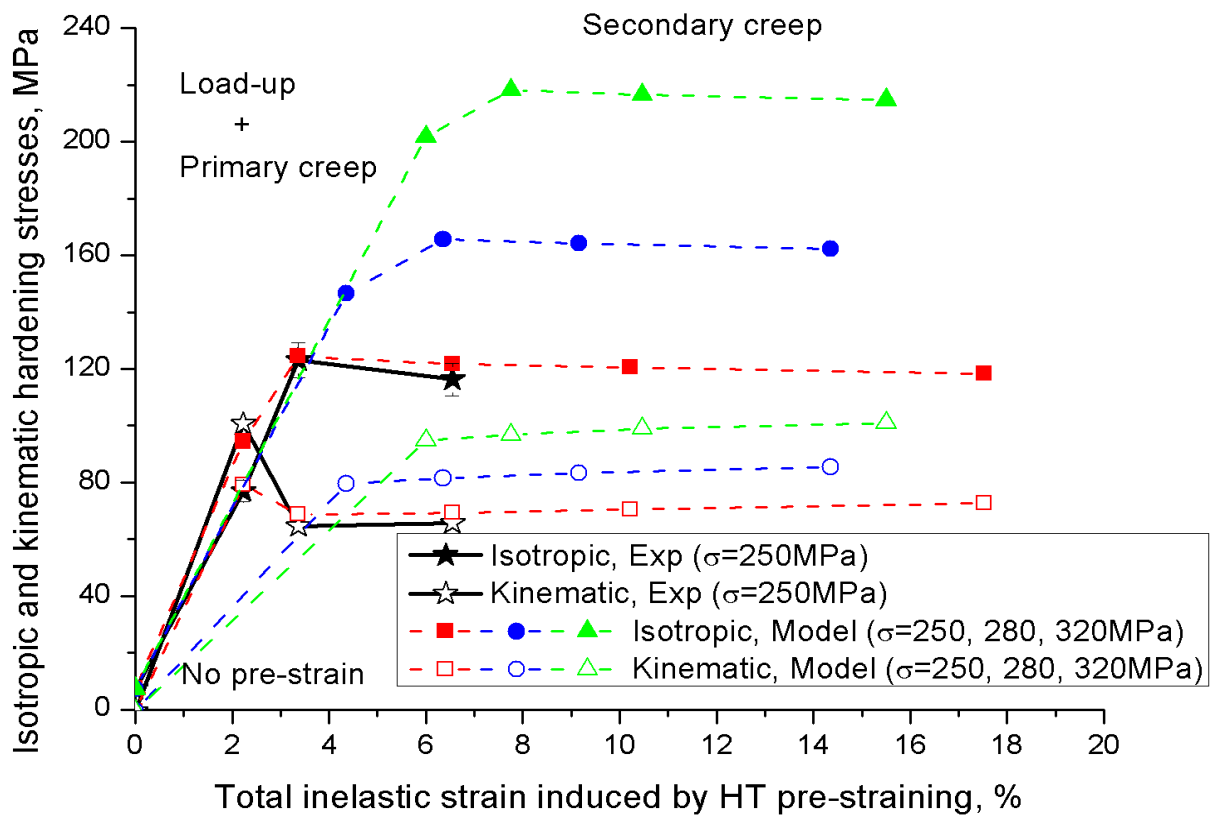

Fig 4. Experimentally determined and model predicted evolution of the empirical isotropic and kinematic stress terms resulted from different stresses over an extended short-term period of creep.

In summary, a physical creep model is derived in this study based on a thermodynamic approach and is combined with a multi-scale self-consistent model to evaluate the effect of creep pre-strain history on the Bauschinger effect of Type $316 \mathrm{H}$ polycrystalline stainless steels. The CRSS develops during primary creep and saturates towards the secondary stage. This results from the evolution of the state variable, i.e. the mean spacing between forest dislocation junctions, towards a steady state. The residual stress state of the individual grains also recovers and saturates towards the secondary stage, reflecting the evolution of plastic incompatibility between grains. Evolution of CRSS and residual stress state can be correlated with that of empirical isotropic and kinematic stress terms. Both terms evolve in a similar manner to the physical quantities. Understanding how the material internal state evolves during creep is vital to further assessing material behaviour during cyclic creep-fatigue loading.

This work was financially supported by EDF Energy and we are grateful to Dr Bo Chen and Professors David Smith and Peter Flewitt, University of Bristol, for helpful discussions and providing experimental data.

[1] K.L. Murty, I. Charit, J Nucl Mater 383(1-2) (2008) 189-195.

[2] S. Sridhar, P. Rozzelle, B. Morreale, D. Alman, Metall Mater Trans A 42A(4) (2011) 871-877. 
[3] B. Chen, P.E.J. Flewitt, D.J. Smith, A.C.F. Cocks, Int Mater Rev 60(1) (2015) 1-29.

[4] O.B. Pedersen, L.M. Brown, W.M. Stobbs, Acta Metall Mater 29(11) (1981) 1843-1850.

[5] J.N. Hu, B. Chen, D.J. Smith, P.E.J. Flewitt, A.C.F. Cocks, Int J Plasticity 84 (2016) 203-223.

[6] J.N. Hu, A.C.F. Cocks, Int J Solids Struct 78-79 (2016) 21-37.

[7] J.N. Hu, B. Chen, D.J. Smith, P.E.J. Flewitt, A.C.F. Cocks, Materials Today: Proceedings. 2(2)

(2015) S424-S433.

[8] J.N. Hu, DPhil Thesis, University of Oxford (2015).

[9] B. Chen, J.N. Hu, P.E.J. Flewitt, D.J. Smith, A.C.F. Cocks, S.Y. Zhang, Acta Mater 67 (2014) $207-$ 219.

[10] B. Chen, J.N. Hu, Y.Q. Wang, S.Y. Zhang, S.V. Petegem, A.C.F. Cocks, D.J. Smith, P.E.J. Flewitt, Acta Mater 85 (2015) 229-242.

[11] P. Ostrom, R. Lagneborg, J Eng Mater-T Asme 98(2) (1976) 114-124.

[12] O. Ajaja, J Mater Sci 21(9) (1986) 3351-3356.

[13] A.J. Ardell, M.A. Przystupa, Mech Mater 3(4) (1984) 319-332.

[14] L.Q. Shi, D.O. Northwood, Acta Metallurgica Et Materialia 41(12) (1993) 3393-3400.

[15] H. Mecking, U.F. Kocks, Acta Metall Mater 29(11) (1981) 1865-1875.

[16] H.J. Frost, M.F. Ashby, Pergamon Press, Oxford, 1982.

[17] M. Rieth, A. Falkenstein, P. Graf, S. Heger, U. Jantsch, M. Klimiankou, E. Materna-Morris, H. Zimmermann, Internal report (2004).

[18] M. Evans, J Mater Sci 44(21) (2009) 5842-5851.

[19] M. Rieth, J Nucl Mater 367 (2007) 915-919.

[20] A.C.F. Cocks, S.P.A. Gill, J.Z. Pan, Adv Appl Mech 36 (1999) 81-162.

[21] O.D. Sherby, J. Weertman, Acta Metall Mater 27(3) (1979) 387-400.

[22] B. Chen, J.N. Hu, Y.Q. Wang, S. Kabra, A.C.F. Cocks, D.J. Smith, P.E.J. Flewitt, J Mater Sci 50(17) (2015) 5809-5816.

[23] E.P. Busso, F.A. McClintock, Int J Plasticity 12(1) (1996) 1-28.

[24] G.P.P. Pun, Y. Mishin, Acta Mater 57(18) (2009) 5531-5542. 